\title{
Therapeutic effect of Botulinum toxin- $A$ in 88 patients with Trigeminal Neuralgia with 14-month follow-up
}

Shuang Li ${ }^{\dagger}$, Ya-Jun Lian ${ }^{*}$, Yuan Chen ${ }^{\dagger}$, Hai-Feng Zhang ${ }^{\dagger}$, Yun-Qing Ma, Cai-Hong He, Chuan-Jie Wu, Nan-Chang Xie, Ya-Ke Zheng and Yi Zhang

\begin{abstract}
Background: We investigated the long-term effects and safety of botulinum toxin-A (BTX-A) for treating trigeminal neuralgia (TN). We also studied long-term maintenance of this therapeutic effect.

Methods: A visual analog scale (VAS) score, pain attack frequency per day, patient's overall response to treatment and side effects during 14-month follow-up were evaluated in 88 patients with TN receiving BTX-A. The primary endpoints were pain severity (assessed by VAS) and pain attack frequency per day. The secondary endpoint was the patient's overall response to treatment, assessed using the Patient Global Impression of Change. The influence of different doses ( $\leq 50,50-100$ and $\geq 100 \mathrm{U}$ ) on the therapeutic effect was evaluated.

Results: Treatment was deemed "effective" within 1 month in 81 patients and at 2 months in 88 patients (100\%). The shortest period of effective treatment was 3 months, and complete control of pain was observed in a maximum of 46 patients. The therapeutic effect decreased gradually after 3 months, and the prevalence of effective treatment at 14 months was 38.6\%, with complete control of pain seen in 22 patients (25\%). There was no significant difference in the prevalence of effective treatment between different dose groups at identical time points $(p>0.05)$. Three patients showed swelling at injection sites and 10 patients showed facial asymmetry, both of which disappeared spontaneously without special treatment.

Conclusion: Local subcutaneous injection of BTX-A for TN treatment has considerable therapeutic effects lasting several months and is safe for this indication. At least one-quarter of patients maintained complete analgesia. The maintenance period of the therapeutic effect may be related to the reduction in the VAS score after the first injection of BTX-A.
\end{abstract}

Keywords: Trigeminal neuralgia; Botulinum toxin A; Long-term therapeutic effect; Subcutaneous injection

\section{Background}

Trigeminal neuralgia (TN) is characterized by sudden (usually unilateral), severe, brief, stabbing, recurrent pain in the distribution of one or more branches of the trigeminal nerve. TN is usually evoked by the stimulation of facial "trigger points", such as teeth-brushing, face-washing, drinking, or shaving. Because the pain is long-term and severe, the quality of life of patients is severely affected [1].

\footnotetext{
* Correspondence: lianyajun840317@163.com

†Equal contributors

Department of Neurology, the First Affiliated Hospital, Zhengzhou University, 1 Jianshe East R, Zhengzhou 450052, Henan Province, People's Republic of
} China

\section{亚 Springer}

Botulinum toxin A (BTX-A) is a type of exotoxin produced by Clostridium botulinum. It relaxes muscles through the release of acetylcholine at nerve-muscle junctions. Therefore, BTX-A is used widely in cosmetology and treating dysmyotonia [2]. BTX-A can also inhibit secretion from glands through inhibition of the release of acetylcholine from cholinergic nerve terminals [3]. It is used in the treatment of bromhidrosis, hyperhidrosis, hygrostomia, vasomotor rhinitis, and rhinorrhea.

Recently, the use of BTX-A for pain management has received considerable attention. In 2002, Micheli et al. were the first to report that BTX-A can relieve TN [4]. Subsequent reports also supported the beneficial effects 
of BTX-A in TN treatment $[5,6]$. The longest observation period of the therapeutic effect of BTX-A in TN is 6 months $[7,8]$. In a review focusing on BTX for the treatment of neuropathic pain, the longest observation period for BTX-A in TN was 4 months [9]. Reports focusing on longer observation periods or factors related to the maintenance of long-term effects are lacking. Here, we report the effect of BTX-A in the treatment of 88 TN patients with a follow-up of 14 months.

\section{Methods}

\section{Ethical approval of the study protocol}

The study protocol was approved by the Ethics Committee of Zhengzhou University (Zhengzhou, China). All patients provided written informed consent to participate in the study.

\section{Patient recruitment}

We conducted an open study. Eighty-eight subjects with primary one-branch classical TN treated with BTX-A were recruited as outpatients or inpatients from the Neurology Department of the First Affiliated Hospital, Zhengzhou University from January 2009 to June 2012. The goal, procedure and safety aspects of the study were explained to each of the patients and Informed consent was included in the documents of each patient. The medications which were used to alleviate their pain were asked to remain unchanged during the course of the study. All patients showed symptom improvement after 8 weeks of treatment, and were followed up for 12 months. And for some patients who didn't show symptom improvement within 2-4 weeks,we gave an additional dose, and plus the additional dose for the first dose as the treatment dose. The patients who showed improvement after 8 weeks of reinjection were followed up for 12 months too. Before hospital admission, all patients underwent laboratory examinations to exclude coagulation disorders and severe dysfunction of the heart, liver or kidneys. Detailed physical examinations as well as CT and MRI of the cranium were conducted to exclude secondary TN.

\section{Therapeutic regimens}

BTX-A crystals (HengLi ${ }^{\circ}$; 100 U/bottle; Lanzhou Bioproduction Institute, Lanzhou, China) was diluted with $0.9 \%$ saline $(2 \mathrm{~mL})$ to $50 \mathrm{U} / \mathrm{mL}$ before use. The BTX-A solution was injected in the facial area and the trigger point of pain at a depth of $0.5 \mathrm{~cm}, 2.5-5$ IU per point, separation of $15 \mathrm{~mm}$ and at $15-20$ injection points. And for some patients the pain within one side of gum, the injection points were $4-10$, and because the gingival mucosa is thinner than skin, the dosage were fewer than facial area in each point.
Observation indices and evaluation of therapeutic effects Information regarding pain (including the extent of pain, pain attack frequency,and side effects) was recorded in detail each day by patients before and after therapy. Clinic visits or telephone follow-up were undertaken by the same person every week for 2 months and every month thereafter. The patient's overall response to treatment was followed up each month after therapy. The extent of pain was evaluated through a visual analog scale (VAS) with 11 points on a ruler of length $10 \mathrm{~cm}$ where 0 denoted "no pain" and 10 denoted "most severe pain". Patients pointed to the representive point of pain on the panel without reading it but instead according to their feelings, and the corresponding pain scores were recorded. We adopted the classification that a reduction in the VAS score of $<50 \%$ after treatment meant that treatment was classified as being "ineffective", $50-70 \%$ as "effective", $>75 \%$ as "significantly effective" and " 0 " meant that pain was "completely controlled". The total effective value was defined as the percentage of patients with a reduction in the VAS score of $\geq 50 \%$. The patient's overall response to treatment assessed using the Patient Global Impression of Change. PCIC is a single-item rating by patients of their improvement with treatment during the therapy on a 7-point scale that ranges from 'very much improved' to 'very much worse' with 'no change' as the mid-point.

\section{Side effects}

Information regarding side effects (time, extent, duration, frequency, treatment, outcomes of treatment, and correlation with treatment) was recorded in detail.

\section{Statistical analyses}

Data are the mean \pm SD and were analyzed using SPSS ver20.0 (SPSS, Chicago, IL, USA). The effective value was analyzed using the $x^{2}$ test. $\mathrm{p}<0.05$ was considered significant.

\section{Results}

General information

The patient cohort comprised 38 males (43.2\%) and 50 females $(56.8 \%)$. The age range was 33-89 years (mean, 60.6 years; $\mathrm{SD}=11.90$ ). Disease progression was $14-150$ months with mean of 52 months and SD of 50.17. All 88 patients showed symptom improvement within 2 weeks. The percentage of patients in whom treatment was effective at 2 months reached $100 \%$.

\section{Distribution of pain area}

The pain area was in the first branch of the trigeminal nerve in 19 cases, in the second branch in 42 cases, and in the third branch in 27 cases. 


\section{Injection dose}

Maximal and minimal doses were $170 \mathrm{U}$ and $25 \mathrm{U}$, respectively. The dose was $\leq 50 \mathrm{U}$ in 43 cases, $50-100 \mathrm{U}$ in 32 cases, and $\geq 100 \mathrm{U}$ in 13 cases.

\section{Therapeutic effect at different time points}

Treatment was classified as being effective within 1 month in 81 cases, and was effective at 2 months in 88 cases (prevalence of effective treatment of $100 \%$ ). All the patients who showed symptoms improvement significantly reduced pain attack frequency. TN was classified as being completely controlled at 3 months in 46 cases. The number of cases in whom treatment was classified as being effective at 3 months decreased to 76 , and the number of cases with a reduction in the VAS score of 50-75\% decreased from 30 to 17 at 2 months. After 3 months, the effect of BTX-A decreased gradually, with a prevalence of effective treatment of $38.6 \%$ at 14 months, and of TN being completely controlled in 22 cases (25\%) (Table 1).More than $90 \%$ patients with effective treatment reported that they were "much improved" or "very much improved" each month (Table 2 ).

\section{Comparison of effective values and pain attack frequency at different doses}

There was no significant difference in the prevalence of effective treatment between different dose groups at identical time points between 1 month and 14 months (p > 0.05) (Table 3).

There was no significant difference in the prevalence of pain attack frequency between different dose groups at identical time points between 1 month and 14 months (p > 0.05) (Table 4).
Doses for patients in whom treatment was effective only at 3 months

The number of patients in whom treatment was effective only at 3 months decreased from 88 to 76 . The 12 patients in whom treatment was effective only for 3 months comprised 4 cases receiving $25 \mathrm{U}, 3$ cases receiving 50 U, 2 cases receiving 75 U, 2 cases receiving $100 \mathrm{U}$, and 1 case receiving $125 \mathrm{U}$.

\section{Side effects}

Systemic side effects were not observed. Local swelling at injection sites occurred in 3 patients and disappeared within 7 days, 1 cases receiving $25 \mathrm{U}, 1$ cases receiving $75 \mathrm{U}, 1$ cases receiving $125 \mathrm{U}$. Muscle relaxation at injection sites occurred in 10 patients and disappeared within 6-8 weeks, 1 case receiving $45 \mathrm{U}, 2$ case receiving $50 \mathrm{U}, 1$ case receiving $70 \mathrm{U}, 2$ case receiving $75 \mathrm{U}, 2$ case receiving $100 \mathrm{U}, 1$ case receiving $120 \mathrm{U}, 1$ case receiving $125 \mathrm{U}$. All side effects were mild and disappeared without the need for further treatment.

\section{Discussion}

BTX-A has been used against neuropathic pain, including post-herpetic neuralgia [10], diabetic neuropathic pain [11], occipital neuralgia [12], TN [13] and chronic facial pain [14]. The present study compared the effects of $75 \mathrm{U}$ BTX-A and placebo in the treatment of primary TN for 8 weeks. BTX-A significantly decreased the VAS score 1 week after BTX-A injection and the effect continued for 8 weeks, suggesting that multiple subcutaneous injections of BTX-A in the TN area can significantly relieve pain [5]. Studies have suggested that injection of BTX-A in an area near the zygomatic arch or masseter muscle can also be effective $[15,16]$.

Table 1 Comparison of the prevalence of effective treatment at different time points

\begin{tabular}{ccccc}
\hline Time (months) & Effective, \% (no.) & Significantly effective, \% (no.) & Completely controlled, \% (no.) & Total effective value, \% (no.) \\
\hline 1 & $31.8(28)$ & $18.2(16)$ & $42.0(37)$ & $92.0(81)$ \\
2 & $34.1(30)$ & $19.3(17)$ & $46.6(41)$ & $100(88)$ \\
3 & $19.3(17)$ & $14.8(13)$ & $52.3(46)$ & $86.4(76)$ \\
4 & $9.1(8)$ & $13.6(12)$ & $50.0(44)$ & $72.7(64)$ \\
5 & $8.0(7)$ & $12.5(11)$ & $50.0(44)$ & $70.5(62)$ \\
6 & $8.0(7)$ & $9.1(8)$ & $46.6(41)$ & $63.6(56)$ \\
7 & $6.8(6)$ & $9.1(8)$ & $42.0(37)$ & $58.0(51)$ \\
8 & $5.7(5)$ & $10.2(9)$ & $37.5(33)$ & $53.4(47)$ \\
9 & $6.8(6)$ & $8.0(7)$ & $37.5(33)$ & $52.3(46)$ \\
10 & $3.4(3)$ & $9.1(8)$ & $37.5(33)$ & $50.0(44)$ \\
11 & $2.3(2)$ & $9.1(8)$ & $36.4(32)$ & $47.7(42)$ \\
13 & $3.4(3)$ & $9.1(8)$ & $31.8(28)$ & $44.3(39)$ \\
14 & $6.8(6)$ & $8.0(7)$ & $25.0(22)$ & $39.8(35)$ \\
\end{tabular}


Table 2 Comparison of the Patient Global Impression of Change (PGIC) in Patients with a reduction in the VAS score of $\geq \mathbf{5 0} \%$ at different time points

\begin{tabular}{|c|c|c|c|c|}
\hline Time (months) & Patients with a reduction in the VAS score of $\geq 50 \%$ (n) & Very much improved $\left(n_{1}\right)$ & Much improved $\left(\mathrm{n}_{2}\right)$ & $\mathrm{n}_{1+} \mathrm{n}_{2} / \mathrm{n} \times 100 \%$ \\
\hline 1 & 81 & 45 & 32 & $95.06 \%$ \\
\hline 2 & 88 & 51 & 32 & $93.18 \%$ \\
\hline 3 & 78 & 60 & 13 & $93.59 \%$ \\
\hline 4 & 64 & 56 & 6 & $96.88 \%$ \\
\hline 5 & 62 & 53 & 8 & $98.39 \%$ \\
\hline 6 & 56 & 46 & 9 & $96.43 \%$ \\
\hline 7 & 51 & 42 & 6 & $94.12 \%$ \\
\hline 8 & 48 & 40 & 7 & $97.92 \%$ \\
\hline 9 & 46 & 39 & 5 & $95.66 \%$ \\
\hline 10 & 44 & 40 & 3 & $97.73 \%$ \\
\hline 11 & 42 & 39 & 2 & $97.62 \%$ \\
\hline 12 & 39 & 36 & 1 & $94.87 \%$ \\
\hline 13 & 35 & 30 & 3 & $94.28 \%$ \\
\hline 14 & 34 & 28 & 6 & $100 \%$ \\
\hline
\end{tabular}

The present study demonstrated that treatment was effective within 4 weeks in 81 cases, and within 4-8 weeks in 7 cases. These findings suggested that observation of $\geq 8$ weeks is necessary for evaluation of therapeutic effects. Five cases showed a decreased VAS score after 2 months and that their pain was completely controlled at 3 months. These results suggest that the therapeutic effect in some cases is slow. Micheli et al. reported that recurrent facial spasm is accompanied simultaneously by recurrent pain after 12 weeks in patients with facial spasms complicated by TN [4]. Therefore, Micheli et al. proposed that the duration of the effect of BTX-A for treating TN is consistent with its relaxation of muscle tension. In general, relaxation of muscle tension by BTX-A can last for 3-6 months. In the present study, the prevalence of effective treatment at 6 months and 14 months was $63.6 \%$ and $38.6 \%$, including 41 cases and 22 cases of completely controlled pain, respectively. In 2012, we reported 1 case of TN without recurrence in 2 years [17], which is beyond the period of relaxation of muscle tension elicited by BTX-A. This finding suggests that BTX-A can relieve pain for longer than it can relax muscle tension. Therefore, we think that the analgesic effect of BTX-A

Table 3 Comparison of the prevalence of effective treatment at different doses

\begin{tabular}{|c|c|c|c|c|}
\hline $\begin{array}{c}\text { Time } \\
\text { (months) }\end{array}$ & $\begin{array}{l}\quad \leq 50 \cup(n=43) \\
\text { Effective value, \% (n) }\end{array}$ & $\begin{array}{c}50-100 \cup(n=32) \\
\text { Effective value, \% (n) }\end{array}$ & $\begin{array}{l}\quad \geq 100 \cup(n=13) \\
\text { Effective value, } \%(n)\end{array}$ & $p$ \\
\hline 1 & $88.4(38)$ & $93.8(30)$ & $100.0(13)$ & 0.360 \\
\hline 2 & 100.0 & $100.0(32)$ & $100.0(13)$ & - \\
\hline 3 & $88.4(38)$ & $87.5(28)$ & $76.9(10)$ & 0.558 \\
\hline 4 & 76.7 (33) & $65.6(21)$ & $76.9(10)$ & 0.528 \\
\hline 5 & $74.4(32)$ & $62.5(20)$ & $76.9(10)$ & 0.459 \\
\hline 6 & $72.1(31)$ & $56.3(18)$ & $53.8(7)$ & 0.270 \\
\hline 7 & $65.1(28)$ & $53.1(17)$ & $46.2(6)$ & 0.376 \\
\hline 8 & $60.5(26)$ & $50.0(16)$ & $38.5(5)$ & 0.337 \\
\hline 9 & $58.1(25)$ & $50.0(16)$ & $38.5(5)$ & 0.437 \\
\hline 10 & $58.1(25)$ & $46.9(15)$ & $30.8(4)$ & 0.203 \\
\hline 11 & $55.8(24)$ & $46.9(15)$ & 23.1 (3) & 0.116 \\
\hline 12 & $51.2(22)$ & $43.8(14)$ & 23.1 (3) & 0.202 \\
\hline 13 & $48.8(21)$ & $37.5(12)$ & $15.4(2)$ & 0.892 \\
\hline 14 & $46.5(20)$ & $37.5(12)$ & $15.4(2)$ & 0.128 \\
\hline
\end{tabular}


Table 4 Comparison of the prevalence of pain attack frequency at different doses

\begin{tabular}{|c|c|c|c|c|}
\hline Time (months) & $\begin{array}{c}\leq 50 \mathrm{U}(\mathrm{n}=43) \\
\text { Attack frequency, time/24 h }\end{array}$ & $\begin{array}{c}50-100 \mathrm{U}(\mathrm{n}=32) \\
\text { Attack frequency, time/24 h}\end{array}$ & $\begin{array}{c}\geq 100 \mathrm{U}(\mathrm{n}=13) \\
\text { Attack frequency, time/24 h }\end{array}$ & $p$ \\
\hline Before treatment & $45.02 \pm 85.56$ & $30.40 \pm 49.93$ & $83.23 \pm 119.49$ & 0.872 \\
\hline 1 & $4.72 \pm 10.05$ & $2.56 \pm 4.58$ & $1.92 \pm 2.96$ & 0.154 \\
\hline 2 & $4.16 \pm 9.49$ & $1.63 \pm 2.65$ & $2.38 \pm 2.87$ & 0.122 \\
\hline 3 & $2.15 \pm 4.07$ & $1.31 \pm 2.39$ & $1.42 \pm 1.78$ & 0.613 \\
\hline 4 & $1.00 \pm 2.14$ & $1.43 \pm 2.69$ & $1.50 \pm 1.90$ & 0.528 \\
\hline 5 & $1.00 \pm 2.17$ & $1.50 \pm 2.74$ & $1.40 \pm 2.12$ & 0.747 \\
\hline 6 & $0.97 \pm 2.15$ & $1.33 \pm 2.61$ & $1.43 \pm 2.44$ & 0.908 \\
\hline 7 & $0.54 \pm 1.04$ & $1.59 \pm 2.92$ & $2.5 \pm 4.18$ & 0.611 \\
\hline 8 & $0.56 \pm 1.05$ & $1.50 \pm 2.78$ & $1.00 \pm 2.24$ & 0.735 \\
\hline 9 & $0.52 \pm 0.96$ & $1.43 \pm 2.76$ & $2.00 \pm 4.47$ & 0.823 \\
\hline 10 & $0.48 \pm 0.96$ & $1.00 \pm 1.60$ & $0.00 \pm 0.00$ & 0.338 \\
\hline 11 & $0.46 \pm 0.98$ & $1.00 \pm 1.60$ & $0.00 \pm 0.00$ & 0.362 \\
\hline 12 & $0.55 \pm 1.01$ & $0.71 \pm 1.20$ & $0.00 . \pm 0.00$ & 0.570 \\
\hline 13 & $0.62 \pm 1.12$ & $0.83 \pm 1.27$ & $2.00 \pm 2.83$ & 0.573 \\
\hline 14 & $0.45 \pm 0.89$ & $0.75 \pm 1.22$ & $1.00 \pm 1.41$ & 0.663 \\
\hline
\end{tabular}

is not related to the inhibition of acetylcholine release in nerve-muscle junctions.

With respect to the dose range, reports have suggested values from $12.5 \mathrm{U}$ to $100 \mathrm{U}$ [9], with the range of 50$100 \mathrm{U}$ seeming to be the best. The dose range used in the present study was $25-170 \mathrm{U}$. The patient mentioned above who remained free of TN for 2 years was the first patient with TN of the third branch that we treated early. The VAS score was 10 and the dose of BTX-A was $170 \mathrm{U}$ (which was the maximum dose we used in all TN patients, she received an additional dose 10 days after the first injection.). At 4 weeks, the VAS score was 0 and, unexpectedly, there was no pain for 3 years.

The present study suggests that there is no significant difference in the therapeutic effect and pain attack frequency between different dose groups at identical time points between 1 month and 14 months. We also showed that effective treatment was observed within 1 month in 81 cases and at 2 months in 88 cases. Effective treatment at 3 months was observed in only 76 cases. That is, therapeutic effect was maintained only for 3 months in 12 patients. The 12 cases showing a therapeutic effect only for 3 months were those using all doses of BTX-A. Patients with a short-term therapeutic effect were mainly those showing a decrease in the VAS score after the first injection. Patients in whom symptoms were completely controlled showed this effect at 3 months (46 cases), of which 14 cases showed no pain at 14 months. These results suggest that the factor influencing the long-term effect of BTX-A is not dose but the extent of the reduction of the VAS score. That is, the more the VAS score decreases, the longer the therapeutic effect is maintained. Therefore, to reach complete control of symptoms and limit the minimal effective dose, we recommend a small dose for the first injection and increase the dose after 2-4 weeks for those in whom complete control of symptoms after the first injection is not observed.

The mechanism of action of BTX-A in pain therapy is not clear. Recent studies suggest that BTX-A can relieve pain through inhibition of the release of cytokines from sensory terminals and by reducing neurogenic inflammation [17]. This effect can be accomplished selectively through inhibition of $\mathrm{C}$-fibers and the transient receptor potential vanilloid (TRPV)1 receptor [18], inhibition of calcitonin gene-related peptide from trigeminal sensory neurons in the brainstem [19], or decrease of peripheral sensitization and central sensitization [20]. In addition, the proposal regarding the existence of BTX-A-sensitive and -insensitive C-fibers and the TRPV1 receptor [18] may explain the different effects observed in different patients.

We observed only mild local side effects. Local swelling at injection sites occurred in 3 patients and disappeared within 7 days ( 1 cases receiving $25 \mathrm{U}, 1$ cases receiving $75 \mathrm{U}$, 1cases receiving $125 \mathrm{U}$ ). Muscle relaxation and facial asymmetry at injection sites occurred in 10 patients and disappeared within $6-8$ weeks ( 1 case receiving $45 \mathrm{U}, 2$ case receiving $50 \mathrm{U}, 1$ case receiving $70 \mathrm{U}, 2$ case receiving $75 \mathrm{U}, 2$ case receiving $100 \mathrm{U}, 1$ case receiving $120 \mathrm{U}, 1$ case receiving $125 \mathrm{U}$ ), which may have been due to the diffusion of BTX-A into deeper layers of facial muscles as well as the induction of transient muscle paralysis. All side effects were mild and disappeared without the requirement for special treatment. 
During the study, we observed more than $90 \%$ patients with effective treatment reported that they were "much improved" or "very much improved" each month. That means the treatment not only improved the TN symptoms, but also showed an improvement in the quality of life, emotional function, side effect burden. So, we think the subcutaneous injection of BTX-A for treating $\mathrm{TN}$ is a clinical trial achieved a good satisfaction out of participants' aggregation all of the components of their experience-pain relief, improvement in physical and emotional functioning, side effects and so on.

And for those patients who have lost the injection efficacy, 10 patients choose the surgical treatment, 4 patients received the medical treatment, and 40 patients received re-injection.

\section{Conclusion}

In conclusion, we found that local subcutaneous injection of BTX-A for treating TN had considerable beneficial effects lasting for several months and was safe to use for this indication. One-quarter of patients could reach complete control of pain for $>14$ months, and maintenance of the therapeutic effect was related to a reduction in the extent of the VAS score after the first injection.

\section{Competing interests}

The authors declare that they have no competing interest.

\section{Authors' contributions}

YJL study concept and design. SL acquisition of data. HFZ carried out the treatment and studies, drafted the manuscript. $Y C$ participated in the sequence alignment, analysis and interpretation. YQM, CHH, CJW revise the manuscript. NCX, YKZ, YC study supervision. All authors read and approved the final manuscript.

\section{Acknowledgements}

The study was supported by the National Natural Science Foundation of China (grant number 81371438), the Provincial Natural Science Research Foundation of the Education Department of HeNan Province (2011A320038) and a project of the HeNan Health Department (201203006).

Received: 5 April 2014 Accepted: 3 June 2014

Published: 22 June 2014

\section{References}

1. Hall GC, Carroll D, Parry D, McQuay HJ (2006) Epidemiology and treatment of neuropathic pain: the UK primary care perspective [J]. Pain 122(1-2):156-162

2. Lian YJ, Wei HL, Zhang BA, Liu HB, Xu YM, Fang SY, Li ZF, Fang GY, Lu H, Jia YJ, Zhao XY, Wei JK, Zhang L, Song B (2009) Treatment of 795 facial spasm and local dystonia with Botulinum toxin A. J Zhengzhou Univ (Med Sci) 44(2):440-442

3. Bowen ME, Weninger K, Bnmger AT (2004) Single molecule observation of liposome-bilayer fusion thermally induced by soluble Nethyl maleimide sensitive factor attachment protein receptors (SNAREs). Biophys J 87:3569-3584

4. Micheli F, Scorticati MC, Raina G (2002) Beneficial effects of botulinum toxin type A for patients with painful tic convulsif. Clin Neuropharmacol 25:260-262

5. Wu CJ, Lian YJ, Zheng YK (2012) Botulinum toxin type A for the treatment of trigeminal neuralgia: results from a randomized, double-blind, placebo-controlled trial. Cephalalgia 32:443-450
6. Shen JH, Lian YJ, Zheng YK, Xie YY, Wei HL (2011) Treatment of primary trigeminal neuralgia with botulinum toxin type A. China J Rehabil Med 26(5):483-484

7. Bohluli B, Motamedi MH, Bagheri SC, Bayat M, Lassemi E, Navi F, Moharamnejad N (2011) Use of botulinum toxin A for drug-refractory trigeminal neuralgia: preliminary report. Oral Surg Oral Med Oral Pathol Oral Radiol Endod 111:47-50

8. Zhu TG, Zhang QL, Luo WF, Mao CJ, Hu WD, Liu CF (2011) Clinical treatment of refractory trigeminal neuralgia with botulinum toxin type $\mathrm{A}$. China Clinical Neuroscience 19(1):32-35

9. Gustavo F, Jose DA, Villanueva P, Asensio-Samper JM (2013) Subcutaneous and perineural Botulinum Toxin Type A For Neuropathic Pain. Clin J Pain [Epub ahead of print]

10. Xiao L, Mackey S, Hui H, Xong D, Zhang Q, Zhang D (2011) Subcutaneous injection of botulium toxin $A$ is beneficial in postherpetic neuralgia. Pain Med 11:1827-1833

11. Yuan RY, Sheu JJ, Yu JM, Chen WT, Tseng IJ, Chang HH, Hu CJ (2009) Botulinum toxin for diabetic neuropathic pain:a randomized double-blind crossover trial. Neurology 72:1456-1478

12. Taylor M, Silva S, Cottrell C (2008) Botulinum toxin type A in the treatment of occipital neuralgia: a pilot study. Headache 48:1476-1481

13. Wu CJ, Lian YJ (2012) Botulium toxin type A for trigeminal neuralgia-we have the prima facie evidence. Cephalagia 32(15):1156-1157

14. Borodic GE, Acquadro MA (2002) The use of botulinum toxin for the treatment of chronic facial pain. J Pain J Pain 3:21-27

15. Turk U, Ilhan S, Alp R, Sur H (2005) Botulinum toxin and intractable trigeminal neuralgia. Clin Neuropharmacol 28:161-162

16. Zuniga C, Diaz S, Piedimonte F, Micheli F (2008) Beneficial effects of botulinum toxin type A in trigeminal neuralgia. Arq Neuropsiquiatr 66:500-503

17. Aoki KR (2005) Review of a proposed mechanism for the antinociceptive action of botulinum toxin type A. Neurotoxicology 26:785-793

18. Gazerani P, Pedersen NS, Staahl C, Drewes AM, Arendt-Nielsen L (2009) Subcutaneous botulinum toxin type A reduces capsaicin-indecd trigeminal pain and vasomotor reactions in human skin. Pain 141:60-69

19. Meng J, Ovsepian SV, Wang J (2009) Activation of TRPV1 mediates calcitonin generelated peptide release, which excites trigeminal sensory neurons and is attenuated by a retargeted botulinum boxin with anti-nociceptive potential. J Neurosci 29:4981-4992

20. Woolf CJ, Costigan M (1999) Transcriptional and posttranslational plasticity and the generation of inflammatory pain. Proc Natl Acad Sci 96:7723-7730

\section{doi:10.1186/1129-2377-15-43}

Cite this article as: Li et al:: Therapeutic effect of Botulinum toxin-A in 88 patients with Trigeminal Neuralgia with 14-month follow-up. The Journal of Headache and Pain 2014 15:43.

\section{Submit your manuscript to a SpringerOpen ${ }^{\odot}$ journal and benefit from:}

- Convenient online submission

Rigorous peer review

- Immediate publication on acceptance

- Open access: articles freely available online

- High visibility within the field

- Retaining the copyright to your article

Submit your next manuscript at $>$ springeropen.com 